\title{
WIDE ESTIMATION OF DYNAMIC PROPERTIES OF VISCOELASTIC MATERIALS USING BAYESIAN INFERENCE
}

\author{
Fernanda Oliveira Balbino, Cíntia Teixeira Préve \\ Federal University of Paraná, Postgraduate Program in Mechanical Engineering, Curitiba, Brazil \\ e-mail: ferbalbino@yahoo.com.br, cintiapreve@gmail.com \\ Marilda Munaro \\ Federal University of Paraná, Postgraduate Program in Engineering and Science of Materials, Curitiba, Brazil \\ e-mail: marilda.munaro94@gmail.com \\ Paulo Justiniano Ribeiro Junior \\ Federal University of Paraná, Department of Statistics, Curitiba, Brazil \\ e-mail: paulojus@ufpr.br \\ Eduardo Márcio de Oliveira Lopes \\ Federal University of Paraná, Postgraduate Program in Mechanical Engineering, Curitiba, Brazil \\ e-mail: eduardo_lopes@ufpr.br
}

\begin{abstract}
The dynamic behavior of a typical viscoelastic material in wide ranges of frequency and temperature is characterized. A four-parameter fractional derivative model was considered in the frequency domain along with the Arrhenius and WLF models, also for including temperature as a source of variation. A Bayesian framework is adopted and inferences on parameters governing the model quantities of interest are based on samples from posterior distributions obtained by Monte Carlo Markov Chain (MCMC) methods. Posterior predictive checks were conducted to ensure the goodness-of-fit of the model. Based on the results we argue that the Bayesian framework allows more complete and suitable inference about dynamic properties of typical viscoelastic materials, as required for broad and sound vibration control actions.
\end{abstract}

Keywords: Bayesian inference, dynamic properties, posterior distribution, vibration control, viscoelastic material

\section{Introduction}

It is necessary to know dynamic properties of viscoelastic materials (VEMs) in order to efficaciously apply them to vibration control devices. A well-established way of describing the dynamic behavior of VEMs is through their complex modulus representation. The complex notation brings together their elastic and dissipative characteristics respectively represented by the real and imaginary moduli. Alternatively, the ratio between the imaginary modulus and the real one, called the loss factor, takes place of the imaginary modulus. These are known as dynamic properties of a VEM, and they are frequency and temperature dependent.

A four-parameter fractional derivative model has proven to be a powerful tool for describing the frequency dependent dynamic behavior of real VEMs used for passive vibration control (Bagley and Torvik, 1986). The temperature dependence can be included either by the WLF equation or Arrhenius equation (Mead, 1999). Thus, these models are largely adopted to characterize dynamic properties of VEMs, once fed by the corresponding experimental data.

The method of least-squares error is usually employed for associating experimental data to modeling. However, this approach does not take into account the uncertainty in identifying 
dynamic properties due to measurement and modeling errors (Gogu et al., 2010). That uncertainty may significantly affect predictions on the performance of viscoelastic devices.

Randomness is a distinctive feature of dynamic properties of VEMs (Hilton, 2003; Scherbakov and Gurvich, 2005). Experimental statistical variability associated with obtaining dynamic properties of VEMs can reach approximately 20\% (Balbino, 2012), and the factors associated with this uncertainty are not fully controllable. Thus, it is important to employ mechanisms for uncertainty propagation and quantification while determining those properties.

The aim of the present work is to apply the Bayesian inference as a way of assessing wide dynamic behavior of the complex modulus of a typical VEM by taking into account its uncertainty. By "wide dynamic behavior" it is meant that the frequency and temperature ranges are such that the dynamic properties of the VEM can be fully described for use in vibration control actions, either by analytical expressions (as shown in Section 3) or by a nomogram (as shown in section 4). The application of the Bayesian inference is intended to incorporate measurement uncertainties into the model parameter estimates by fitting probability distributions to the parameters of concern. Altogether, that should supply a more detailed and complete (wide) estimation of VEM dynamic properties.

In the past few years, some researchers used the Bayesian approach to identify VEM parameters. Gogu et al. (2010) compared the least-squares method, widely used to identify elastic constants from experimental data, with the Bayesian method. Using a simple three-bar truss example, it was possible to note that the following features revealed themselves systematically better for the Bayesian statistical approach over the basic least-squares method: 1) different sensitivity of the response components to parameters to be identified; 2) different uncertainty in measurements; and 3) high correlation among response components. The authors stated that the main advantage of the Bayesian approach was that it identified the properties probability density function, allowing narrow safety margins and improved uncertainty knowledge on the material properties.

Yet in a probabilistic framework, Zhang et al. (2013) identified Young's modulus of a polymer core in a laminated structure using the Bayesian inference. Their conclusions highlighted that the main advantage was the capacity to return the quantified uncertainty in the posterior probability density function for Young's modulus. However, those studies considered conditions of variable frequency and constant temperature. Hernández et al. (2017) used the Bayesian approach to achieve posterior probability distributions of shear modulus parameters for a thermorheologically simple viscoelastic material. This work considered temperature as a source of variance, although in a limited scope and eithout specifying the number of experimental repetitions.

According to Nashif et al. (1985), temperature is one of the most important external factors affecting dynamic properties of VEMs. In fact, it can be argued, in line with Jones (2001), that it is the most striking. Therefore, a more complete and reliable dynamic characterization must cover wide ranges of frequency and temperature to fully determine the behavior of VEMs under those variables.

As working environments with marked changes in temperature are common in passive vibration control cases, the temperature dependence is also regarded in detail herein along with the frequency dependence. Besides, the posterior predictive check (PPC) (Gelman, 2003) is introduced and applied to assess whether or not the approach leads to a satisfactory description of the observed data, collected through an experimental trial. The same procedure of the PPC is also used in a companion paper to estimate dynamic properties of a VEM and to further design and assess the performance of vibration control devices (Préve et al., 2020). That is another distinctive and important feature which is not covered in the previous related papers. 


\section{Complex modulus representation}

As anticipated, a well-known way of describing dynamic behavior of viscoelastic materials is the representation in terms of complex moduli (Nashif et al., 1985). The real and imaginary parts represent the elastic and dissipative characteristics, respectively. Thus, complex Young's modulus $\bar{E}$ of a viscoelastic material (VEM) can be written as

$$
\bar{E}=E_{R}+\mathrm{i} E_{I}
$$

The corresponding loss factor $\eta_{E}$ is defined as the ratio between the imaginary modulus, $E_{I}$ (also called the "loss modulus"), and the real modulus, $E_{R}$ (also called the "storage modulus"), as

$$
\eta_{E}=\frac{E_{I}}{E_{R}}
$$

Therefore, the loss factor is associated with mechanisms by which the material dissipates energy; the higher the energy dissipation, the higher the loss factor. Thus, complex Young's modulus can also be written as

$$
\bar{E}=E_{R}\left(1+\mathrm{i} \eta_{E}\right)
$$

As a complex quantity, complex Young's modulus can also be described in terms of the modulus and argument as

$$
\bar{E}=E \mathrm{e}^{\mathrm{i} \theta_{E}}
$$

where $E$ is the modulus of $\bar{E}$, and $\theta_{E}$ the argument, respectively, given by

$$
E=\sqrt{E_{R}^{2}+E_{I}^{2}} \quad \theta_{E}=\arctan \frac{E_{I}}{E_{R}}
$$

Due to the aforementioned frequency and temperature dependence, the complex modulus $\bar{E}$ (in $\mathrm{Pa}$ ) should be expressed as

$$
\bar{E}(\omega, T)=E_{R}(\omega, T)\left[1+\mathrm{i} \eta_{E}(\omega, T)\right]
$$

where $\omega$ stands for frequency (in $\mathrm{rad} / \mathrm{s}$ ) and $T$ stands for temperature (in $\mathrm{K}$, or ${ }^{\circ} \mathrm{C}$ ). Models representing the frequency and temperature dependence are presented in the following Sections.

Analogous equations hold for the complex shear modulus.

\section{Four-parameter fractional derivative model}

Fractional derivative models are consistent and efficient in describing dynamic behavior of various VEMs, particularly those used in vibration control (Pritz, 1996; de Sousa et al., 2017). Besides, adequately describing the behavior of VEMs, fractional derivative models are very convenient for engineering calculus in the frequency domain, and the frequency is a variable of concern.

According to Bagley and Torvik (1986), the generalized model of a fractional order, which gives a one-dimensional constitutive equation for VEMs, is given by

$$
\sigma(t)+\sum_{m=1}^{M} b_{m} D^{\beta_{m}}[\sigma(t)]=E_{0} \varepsilon(t)+\sum_{n=1}^{N} E_{n} D^{\alpha_{n}}[\varepsilon(t)]
$$


where $b_{m}, \beta_{m}, E_{0}, E_{n}$, and, $\alpha_{n}$ are real parameters; $\sigma(t)$ and $\varepsilon(t)$ are time histories of stress and strain; and $D^{\alpha_{n}}$ and $D^{\beta_{m}}$ are derivative operators of a fractional order, as per the Riemann-Liouville (RL) definition of which is

$$
D^{\alpha}[x(t)]=\frac{1}{\Gamma(1-\alpha)} \frac{d}{d t} \int_{0}^{t} \frac{x(\tau)}{(t-\tau)^{\alpha}} d \tau
$$

where $\alpha$ is the fractional derivative order, such that $0<\alpha<1, \Gamma$ is the gamma function, and $\tau$ is an integration variable (Bagley and Torvik, 1986; Medeiros et al., 2019).

Following the theoretical application in Bagley and Torvik (1986) for Eq. (3.1), taking $m=n=1, \beta_{1}=\alpha_{1}=\beta$, and $b_{1}=b$ and then applying the Fourier transform to it, a suitable model for complex Young's modulus of a viscoelastic material in the frequency domain with four material parameters is obtained. It is

$$
\bar{E}(\omega)=\frac{\bar{\sigma}(\omega)}{\bar{\varepsilon}(\omega)}=\frac{E_{L}+E_{H} b(\mathrm{i} \omega)^{\beta}}{1+b(\mathrm{i} \omega)^{\beta}}
$$

where $E_{L}\left(=E_{0}\right)$ is the elastic modulus (in Pa), $E_{H}\left(=E_{1} / b\right)$ is the glassy modulus (in Pa), $b$ is a real parameter (in $\mathrm{s}^{\beta}$ ), and $\beta$ is the (dimensionless) order of the associated fractional derivative, so that, as anticipated, $0<\beta<1$. To ensure the thermodynamic constraints of the model, it follows that $E_{H}>E_{L}>0$ and $b>0$. The four model parameters are then, $E_{L}, E_{H}$, $b$, and $\beta$.

Equation (3.2) can be written in terms of its real and imaginary parts (in Pa), generating the following equations

$$
\begin{aligned}
& E_{R}(\omega)=\frac{E_{H}\left(b \omega^{\beta}\right)^{2}+\left(E_{H}+E_{L}\right) \cos (\beta \pi / 2) b \omega^{\beta}+E_{L}}{\left(b \omega^{\beta}\right)^{2}+2 \cos (\beta \pi / 2) b \omega^{\beta}+1} \\
& E_{I}(\omega)=\frac{\left(E_{H}-E_{L}\right) \sin (\beta \pi / 2) b \omega^{\beta}}{\left(b \omega^{\beta}\right)^{2}+2 \cos (\beta \pi / 2) b \omega^{\beta}+1}
\end{aligned}
$$

By Eqs. (2.2) and (3.3), the (dimensionless) loss factor follows as

$$
\eta_{E}(\omega)=\frac{E_{I}(\omega)}{E_{R}(\omega)}=\frac{\left(E_{H}-E_{L}\right) \sin (\beta \pi / 2) b \omega^{\beta}}{E_{H}\left(b \omega^{\beta}\right)^{2}+\left(E_{H}+E_{L}\right) \cos (\beta \pi / 2) b \omega^{\beta}+E_{L}}
$$

\section{Frequency-temperature superposition principle}

In a typical experiment for collecting data on dynamic properties of VEMs, after testing a material sample at each intended temperature in a given frequency range, a set of curves is obtained. For a large number of materials of concern, those curves can be superimposed by the use of the frequency-temperature superposition principle to form a complete characterization in both frequency and temperature (Nashif et al., 1985; Jones, 2001).

This principle is based on the observation that the effects of frequency and temperature on the dynamic properties are qualitatively inverse, so that scaled changes in frequency can correspond to inverse changes in temperature. It then states that, for thermorheologically simple materials, the dynamic properties can be expressed in terms of a unique compound variable - called the "reduced frequency" - which combines the aforementioned effects (Jones, 2001).

Thus, the experimental curves along the frequency, at distinct temperatures, can be superimposed at a unique reference temperature by appropriate shifts in the frequency, giving a rise to a single master curve for each dynamic property. The associated expressions are

$$
E_{R 0}\left(\omega_{R}\right)=\frac{T_{0} \rho_{0}}{T \rho} E_{R}(\omega, T) \quad\left(\eta_{E}\right)_{0}\left(\omega_{R}\right)=\eta_{E}(\omega, T)
$$


where $T_{0}$ is the reference temperature (in $\mathrm{K}$ ), $\rho$ is density (in $\mathrm{kg} / \mathrm{m}^{3}$ ), $\rho_{0}$ is density at the reference temperature (in $\mathrm{kg} / \mathrm{m}^{3}$ ), $\omega_{R}=\alpha_{T}(T) \omega$ is the so-called "reduced frequency" (in $\mathrm{rad} / \mathrm{s}$ ), and $\alpha_{T}$ is the (dimensionless) shift factor which depends on temperature. Its values are: $0<\alpha_{T}<1$ for $T>T_{0}, \alpha_{T}=1$ for $T=T_{0}$ and $\alpha_{T}>1$, for $T<T_{0}$. Subscript 0 refers to a quantity at the reference temperature.

The above expressions establish that, except for the (negligible) factor given by $\left(T_{0} \rho_{0} / T \rho\right)$ for the real modulus, the dynamic properties in a frequency $\omega$ and at a temperature $T$ are equal to the dynamic properties in a compound frequency $\omega_{R}$, in the reference temperature $T_{0}$. Each $\alpha_{T}(T)$ yields a change in the frequency equivalent to the change in temperature from $T$ to $T_{0}$. Thus, the variable frequency and temperature are replaced by the single compound variable $\omega_{R}$, the reduced frequency.

The appropriate description of the shift factor $\alpha_{T}$ is very important during dynamic characterization. The empirical equation of Williams-Landel-Ferry (WLF) and the theoretical Arrhenius equation proved to be appropriate models representing the shift factor (Mead, 1999). However, the Arrhenius equation has the advantage of being more straightforward in its application, considering that it has one parameter less, which justifies its use in the present work. It is given by

$$
\log _{10} \alpha_{T}(T)=T_{A}\left(\frac{1}{T}-\frac{1}{T_{0}}\right)
$$

where $T_{A}$ is the so-called "activation energy" (in $\mathrm{K}$ ) to be determined for each material.

After consolidating various experimental curves into two master curves, the dynamic properties can be presented in a plot called the "reduced frequency nomogram" (Jones, 2001), as in Fig. 1.

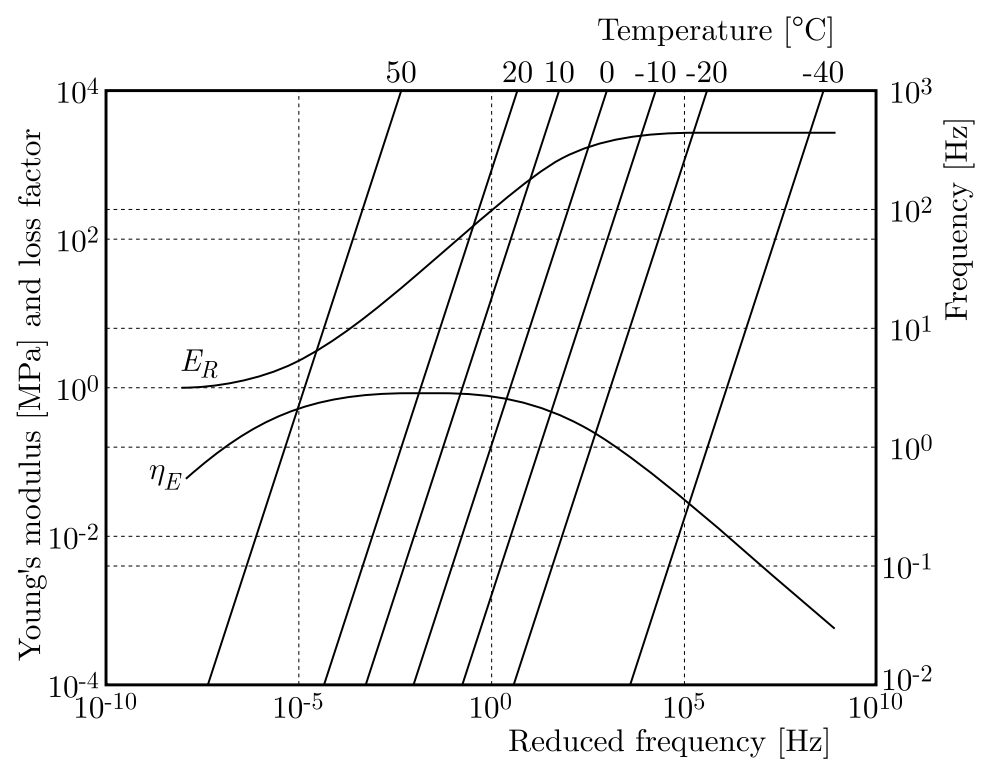

Fig. 1. Reduced frequency nomogram

Accordingly, complex Young's modulus of Eq. (3.2) can be rewritten as follows

$$
\bar{E}(\omega, T)=\frac{E_{L}+E_{H} b\left[\mathrm{i} \alpha_{T}(T) \omega\right]^{\beta}}{1+b\left[\mathrm{i} \alpha_{T}(T) \omega\right]^{\beta}}
$$

As Eq. (4.3) includes the shift factor, the parameters associated with the chosen equation for that factor must subsequently be included in the dynamic characterization of the material of concern. As detailed in the next Section, by considering the Arrhenius equation, there is one 
additional parameter to join the four of the four-parameter fractional derivative model. Then, a total of five parameters are to be estimated in the Bayesian inference.

\section{Bayesian inference}

The Bayesian approach to inference and prediction (Migon et al., 2015) supplies a posterior probability distribution function for model parameters. It allows quantification of uncertainties about a vector of parameters $\theta$, which is unknown, and propagating such uncertainties to the prediction process. A prior probability distribution function for the entire parameter vector of parameters $\theta$ is specified and represents the previous/current knowledge on the parameters in the model. Data is accounted by a likelihood function, which is based on modelling assumptions for the observed random variable $Y$ and describes the likelihood of the observed values $y$ for each given value of the vector parameter $\theta$. By the observed values it is meant, in the current context, the experimental data.

The posterior distribution is obtained by the Bayes rule (Migon et al., 2015) and given by

$$
p(\theta \mid y)=\frac{p(\theta, y)}{p(y)}=\frac{p(\theta) p(y \mid \theta)}{p(y)}
$$

where $p(\theta \mid y)$ represents the posterior distribution function, that is, the distribution of $\theta$ after the values of $Y$ have been observed, $p(y \mid \theta)$ represents the likelihood function which incorporates the information contained in the observed data, $p(\theta)$ represents the so-called "prior distribution function", and the predictive distribution function is denoted by $p(y)$. When the likelihood function is discrete, $p(y)=\sum_{\theta} p(\theta)(y \mid \theta)$; when it is continuous, $p(y)=\int p(\theta) p(y \mid \theta) d \theta$.

The following Sections detail the specific functions of the Bayesian approach and also report as they are regarded in the current work.

\subsection{The likelihood function}

The general Gaussian model for the likelihood function is

$$
Y=f(\text { temperature, frequency, } \boldsymbol{\Theta})+\varepsilon
$$

and it is built by assuming that the errors are independent and normally distributed. In the above expression, $\varepsilon N\left(0, \sigma^{2}\right)$ and $Y$ is the observable response variable, which is updated with the experimental data.

The function $f(\cdot)$ represents the model for complex Young's modulus, and the vector $\Theta$ is equal to $\left[E_{L}, E_{H}, b, \beta, T_{A}\right]$ herein. Computational implementation to be detailed in Section 5.3 is based on the absolute value of the complex modulus, as presented in Eq. $(2.5)_{1}$, i.e., the square root of the sum of the squares of the real and imaginary elasticity moduli. This alternative combines the observed values for the real modulus and the imaginary modulus into single real quantities.

\subsection{Prior distribution}

Weakly informative prior (WIP) distributions were considered for each parameter. WIP distributions provide the benefit of prior information while avoiding the risk of using information that does not exist. WIP distributions change with the sample size because the model should have enough prior information to learn from the data, but the prior information must also be weak enough to learn from the data.

There was available information in previous studies using the same viscoelastic material (Jones, 1992; Lopes 1998) and also in the nomogram provided by the VEM manufacturer. That 
was used to characterize the central tendency and reasonable ranges of the parametric space for the specified prior distributions. However, it is stressed that those studies did not provide information about the variability of each parameter. After incorporating the previous knowledge and running simulations and sensitivity analysis of different setups by respecting the parametric space, independent prior distributions for five parameters, namely, $E_{L}, E_{H}, b_{0}, \beta$ and $T_{A}$, were specified. Some specific remarks in that regard are provided below.

For the parameter $E_{L}$, a truncated normal distribution was chosen considering the restriction that it is always smaller than $E_{H}$. This, in turn, requires no restriction. The parameter $b_{0}$ always assumes very small positive values, thus a lognormal distribution was assigned to ensure that restriction. For the parameter $\beta$, the truncated normal distribution restricted in the interval $[0,1]$ was chosen as the best fit distribution. Finally, a normal distribution with no interval restrictions was assumed for the parameter $T_{A}$. Table 1 summarizes the chosen prior distribution for each parameter.

Previous simulations supplied satisfactory results using the distributions assigned for all the parameters, along with the pertinent restrictions. As the variability of the parameters was the absent information in the previous works and WIP distributions were taken into account, all the variances considered in the study were very high, as seen in Table 1 . That was made to flatter the prior distributions and avoid providing information that does not exist. It is essential to highlight that the previous information about the mean and parametric spaces is combined with a broader variance characterized each WIP distribution.

Table 1. Prior distributions

\begin{tabular}{|c|c|}
\hline $\begin{array}{c}\text { Parameter } \\
\text { of concern }\end{array}$ & Prior distribution \\
\hline \hline$E_{L}[\mathrm{MPa}]$ & Truncated normal $\left(\mu_{E_{L}}, \sigma_{E_{L}}^{2}\right), E_{L} \in(0,10)$, where $\mu_{E_{L}}=5$ \\
& and $\sigma_{E_{L}}^{2}=1000000$ \\
\hline$E_{H}[\mathrm{MPa}]$ & Normal $\left(\mu_{E_{H}}, \sigma_{E_{H}}^{2}\right)$, where $\mu_{E_{H}}=2395$ and $\sigma_{E_{H}}^{2}=1000000$ \\
\hline$b\left[\mathrm{~s}^{\beta}\right]$ & Lognormal $\left(\mu_{\ln (b)}, \sigma_{\ln (b)}^{2}\right)$, where $\mu_{\ln (b)}=0.004$ and $\sigma_{\ln (b)^{2}}=1000000$ \\
\hline$\beta$ & Truncated normal $\left(\mu_{\beta}, \sigma_{\beta}^{2}\right), \beta \in(0,1)$, where $\mu_{\beta}=0.4$ and $\sigma_{\beta}^{2}=1000000$ \\
\hline$T_{A}[\mathrm{~K}]$ & Normal $\left(\mu_{T_{A}}, \sigma_{T_{A}}^{2}\right)$, where $\mu_{T_{A}}=8000$ and $\sigma_{T_{A}}^{2}=1000000$ \\
\hline
\end{tabular}

\subsection{Posterior distribution}

Posterior distributions is usually not obtained in a closed analytical form. In this case, stochastic simulation methods are considered (Gamerman and Lopes, 2006).

Posterior distribution for each parameter were approximated via MCMC techniques using the RJAGS package (Plummer, 2014) in the statistical computing environment R (R Core Team, 2015). The RJAGS package allows the R program to communicate with JAGS (Just Another Gibbs Sampler) software developed by Plummer (2003) to implement the Bayesian Inference using MCMC.

The MCMC sampling algorithm is highly flexible and able to sample from non-linear and complex models with distributions for which analytical results and other sampling methods could not be feasible. This technique performs a random walk over the parametric space followed by a criterion of acceptance-rejection driven by the likelihood function, which guarantees converging results. After a specified number of iterations, a stationary posterior distribution can be reached (Kruschke, 2015).

Through diagnostic plots and other measures, it is possible to check the representativeness, precision and efficiency of the MCMC (Kruschke, 2015). The following plots and measures were 
used to verify the representativeness of the chains herein: a) the trace plot and the density plot; b) the Gelman-Rubin diagnostics (Gelman and Rubin, 1992), as a numeric estimate to verify convergence of the chains; and c) the autocorrelation function to access precision of the chains. It is worth mentioning that the efficiency depends on the choice of a good model and computational power.

After determining the prior distribution and likelihood function, the MCMC was simulated by 102000 iterations with a burn-in period of 1000 iterations. Three chains were generated each time with all the information being stored. More details about MCMC methods and diagnostics can be found in Gamerman and Lopes (2006).

\subsection{Inference on model parameters}

The posterior distribution for each parameter of concern is obtained as the result of a Bayesian inference combining the prior distribution and likelihood function of the parameters. The prior distribution and likelihood function are presented in Sections 5.1 and 5.2.

As presented in Section 5.1, the response variable associated with the likelihood function in Eq. (5.2) corresponds to the observable response variable, which is updated with the observed experimental data obtained for complex Young's modulus, as it will be detailed in Section 6 . This modulus is given herein by Eq. (4.3), which includes the fractional derivative model of Eq. (3.2) and the Arrhenius equation given by Eq. (4.2). As JAGS program does not accept imaginary numbers or any complex numbers in its environment, complex Young's modulus, as presented in Sections 2 and 3, can be represented by its absolute value, Eq. $(2.5)_{1}$, being the real part given by Eq. (3.3) 1 and the imaginary part by Eq. (3.4) 2 .

The aforementioned equations were used to estimate the likelihood function of Eq. (5.2), presented in Section 5.1. It was progressively updated with the observed experimental data, along with the prior distributions of Section 5.2, estimate the parameters $\left[E_{L}, E_{H}, b, \beta, T_{A}\right]$ (of the fractional derivative model and the Arrhenius equation), resulting in a posterior distribution for each parameter (and not a single value), as described in Section 5.3.

\subsection{Posterior Predictive Check (PPC)}

The PPC is a way to check whether or not the fitted model succeeds in describing the observed data. For a Bayesian approach, Kruschke (2015) suggests that the PPC should be fully Bayesian. In that context, the parameter-simulated data generated by the posterior distributions are expected to describe the values of the observed data.

The procedure applied in the current work was introduced by Kruschke (2015), and it is similar to a null hypothesis test. Starting with a hypothesis about the parameter values, simulated data is then generated as if the intended experiment have been repeated many times. The results indicate whether or not the actual data belongs to the space of the simulated data.

The results of the posterior distributions of the parameters simulated by MCMC were used to recreate the simulations, now running 2000 values for each treatment (pair of frequency and temperature) for the real and imaginary modulus and for the absolute value (ABSV). These new results were used to verify the goodness-of-fit of the model.

\section{Experimental data}

The dynamic properties were raised with the aid of the equipment NETZSCH DMA $242 \mathrm{C}$ (Netzsch, 2015). A typical viscoelastic material - commercially known as ISODAMP C-1002 and manufactured by EAR Aearo Technologies LLC (3M Group) - was employed in the tests. 
A total of 25 sample units were tested. The dimensions of each sample unit were $10 \mathrm{~mm}$ long, $4 \mathrm{~mm}$ wide, and $1.37 \mathrm{~mm}$ thick. Previous experiences on the characterization of viscoelastic materials, drawn from Jones $(1992,2001)$ and Lopes (1998), guided the selection of 10 frequencies: $0.1 \mathrm{~Hz}, 0.2 \mathrm{~Hz}, 0.5 \mathrm{~Hz}, 1 \mathrm{~Hz}, 2 \mathrm{~Hz}, 5 \mathrm{~Hz}, 10 \mathrm{~Hz}, 20 \mathrm{~Hz}, 50 \mathrm{~Hz}$, and $100 \mathrm{~Hz}$ (that is from $0.628 \mathrm{rad} / \mathrm{s}$ to $628 \mathrm{rad} / \mathrm{s})$.

The temperatures were also chosen based on previous experiences and on the manufacturer's nomogram. They were: $-40^{\circ} \mathrm{C},-20^{\circ} \mathrm{C},-10^{\circ} \mathrm{C}, 0^{\circ} \mathrm{C}, 10^{\circ} \mathrm{C}, 20^{\circ} \mathrm{C}$, and $50^{\circ} \mathrm{C}$ (that is, from $233 \mathrm{~K}$ to $323 \mathrm{~K}$ ). Smaller intervals were taken around $0^{\circ} \mathrm{C}$ as that was the material transition range (Jones, 1992).

During the test, each thin sample unit is attached to a clamping device and placed inside a thermal chamber of the equipment, at a given temperature. It is then statically preloaded in tension and subjected to a harmonic excitation by an oscillator at the desired frequency. The lower end of the sample is held in place, whereas the upper end is clamped to the oscillator push rod. All the tests are conducted within the linear range.

The harmonic force (input signal) gives rise to deformation of the viscoelastic sample (output signal). This is associated to a harmonic, but phase shifted, displacement. The applied force and the corresponding displacement are measured by internal sensors of the equipment and they are recorded.

These recordings, along with the dimensions of the sample unit, are supplied to the equipment software which computes complex Young's modulus, via Fourier analysis, by

$$
E=\frac{\text { applied force }}{\text { displacement }} \cdot \frac{\text { sample length }}{\text { sample cross section }}
$$

The real and imaginary parts of the complex modulus as well as the loss factor are also computed by the software. These sets of experimental values are considered in Eq. (5.2) to update the response variable $Y$ and allow the parameters in the vector $\boldsymbol{\Theta}$ to be determined. It is noted that the imaginary part of Young's modulus results from the phase shift between the applied force and the displacement which, in turn, is due to damping of the sample unit. Figure 2 shows a sample unit in the clamping device of the equipment just above its thermal chamber.

\section{Results and discussion}

Table 2 shows posterior distributions of the model parameters and the corresponding summaries with posterior means and $95 \%$ credible intervals. All of them are symmetric with and an approximately Gaussian shape, as seen in Fig. 3.

Table 2. Predicted means and 95\% HDI of the estimated parameters

\begin{tabular}{|c|c|c|c|}
\hline \multirow{2}{*}{ Parameter } & \multirow{2}{*}{ Mean } & \multicolumn{2}{|c|}{$95 \%$ HDI } \\
\cline { 3 - 4 } & & Minimum & Maximum \\
\hline \hline$E_{H}[\mathrm{MPa}]$ & 2580 & 2530 & 2630 \\
\hline$E_{L}[\mathrm{MPa}]$ & 4.77 & 4.71 & 4.83 \\
\hline$b\left[\mathrm{~s}^{\beta}\right]$ & 0.00421 & 0.00413 & 0.00429 \\
\hline$\beta$ & 0.393 & 0.390 & 0.396 \\
\hline$T_{A}[\mathrm{~K}]$ & 9770 & 9690 & 9850 \\
\hline
\end{tabular}

MCMC diagnostics was performed in order to ensure that the chains had indeed converged to the posterior distributions (Kruschke, 2015). Figure 4 shows an example of the aforementioned convergence performed to the parameter $E_{H}$. That diagnostics was performed for all the 


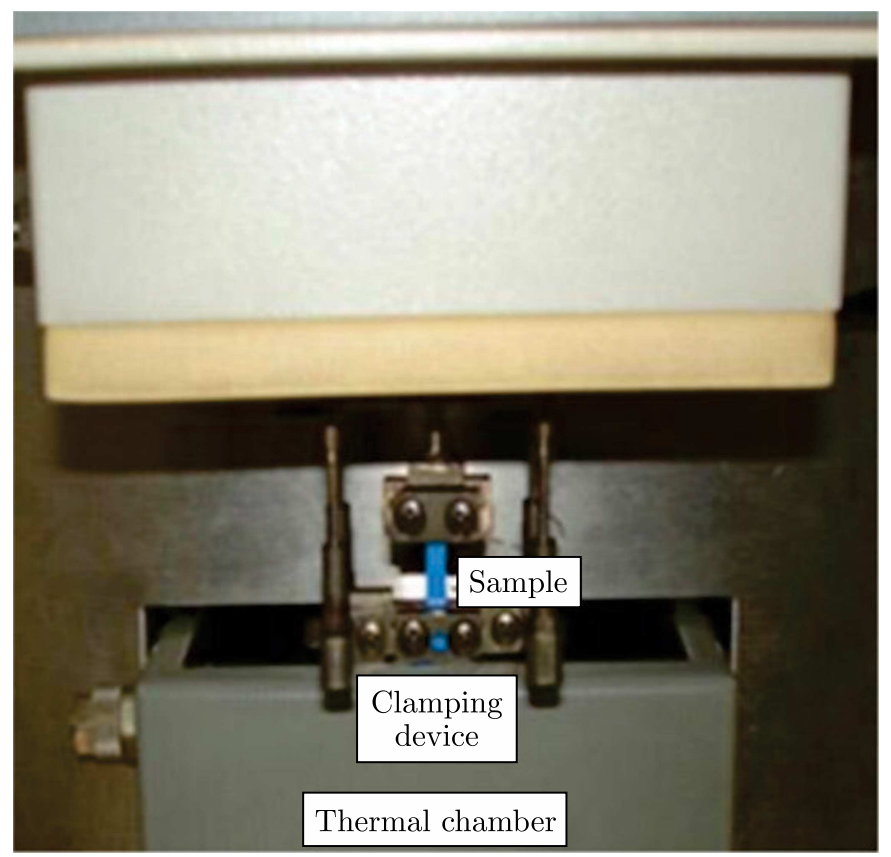

Fig. 2. Sample unit of VEM in the equipment
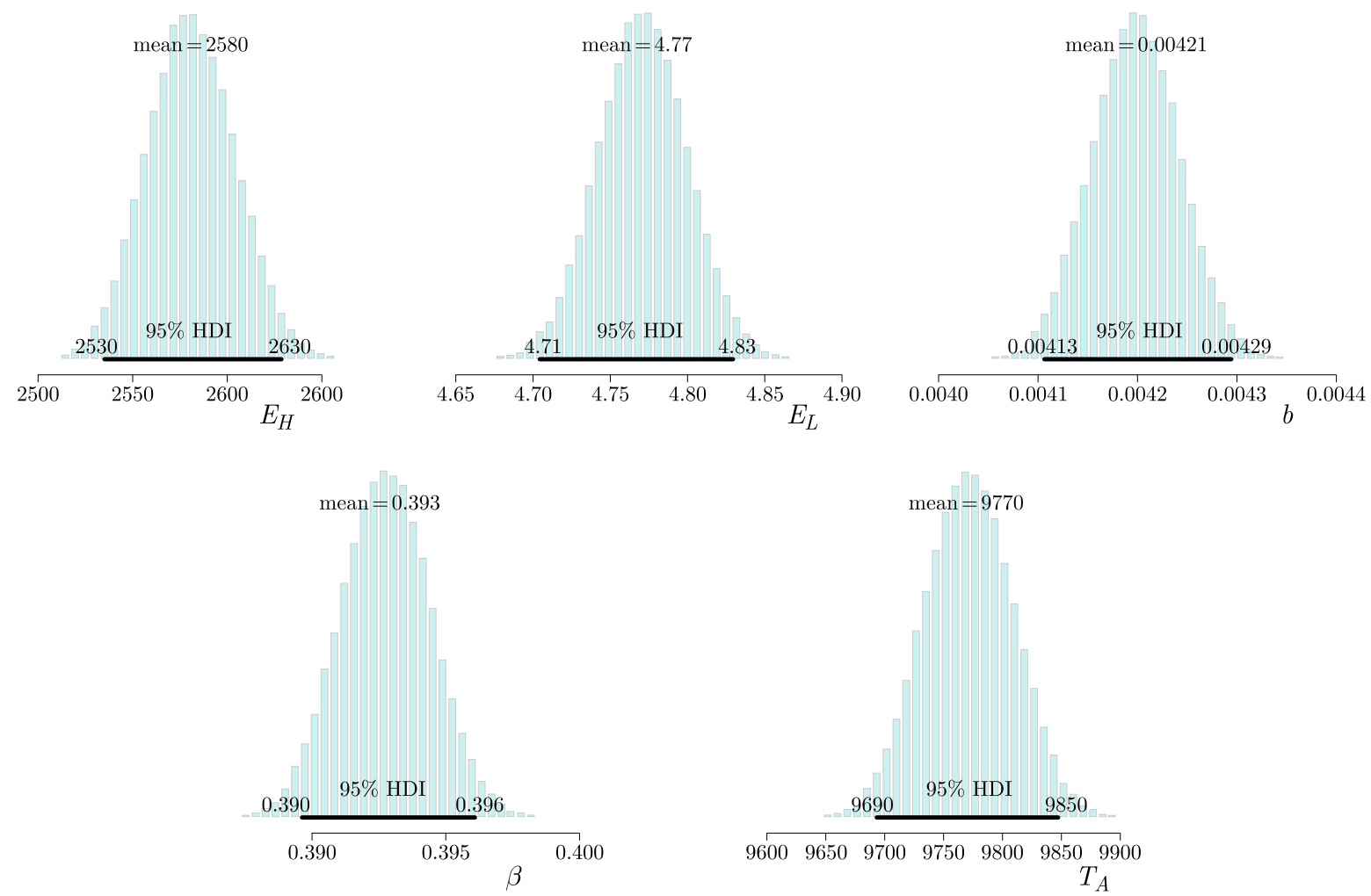

Fig. 3. Posterior probability density functions of the parameters

estimated parameters to check for convergence. That is a good practice which should be always carried out and reported.

By the trace plot, in panel (a) of Fig. 4, it is shown that the chains are superimposed in the same region of the parametric space leading to convergence between the chains. In the density plot, of panel (d), overlapping intervals are averaged to produce a smooth representation of 
(a)

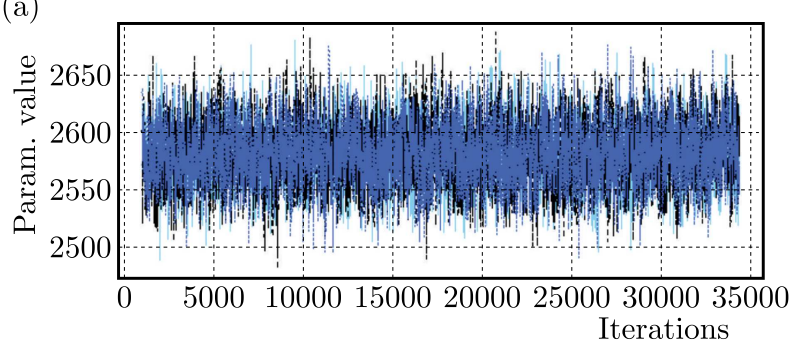

(c)

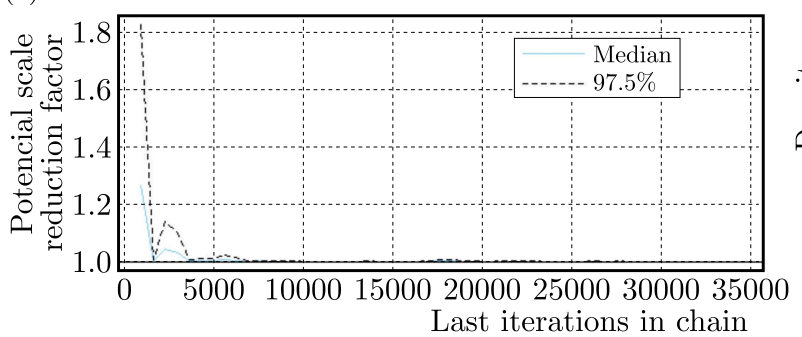

(b)

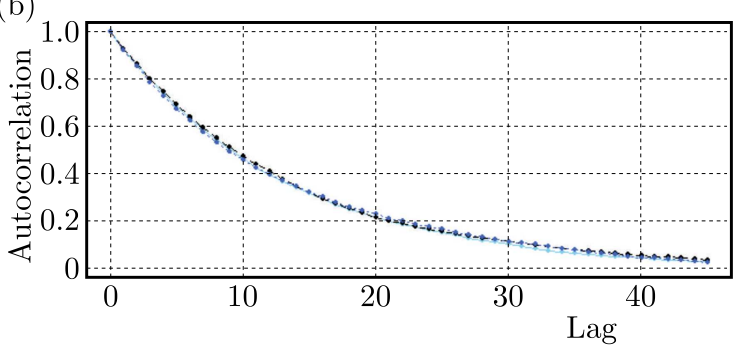

(d)

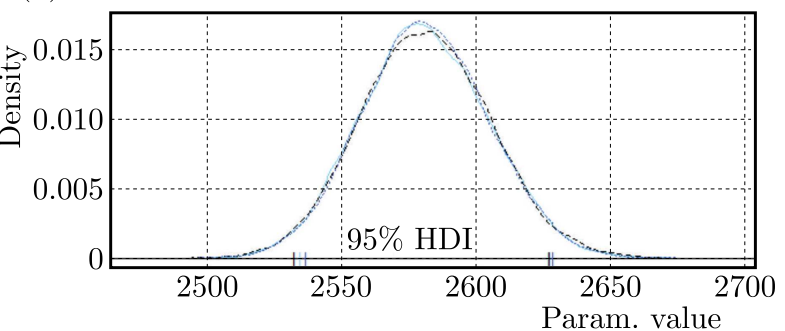

Fig. 4. Convergence diagnostics performed for the parameter $E_{H}$ : (a) trace plot,

(b) autocorrelation function, (c) Gelman-Rubin estimation, (c) density plot

the probability density. For a graph of two or more chains, they should overlap as a sign that representative values of the posterior distribution are produced. It can also be checked the $95 \%$ HDI, which for different chains must have only slightly different values as they are finite samples of the posterior distribution.

The Gelman-Rubin estimation, exhibited in panel (b), is one of the numerical estimates proposed to verify representativeness (Gelman and Rubin, 1992). This method has the premise of verifying the variance between the chains generated in relation to the variance within the chains. Therefore, if all chains are established in the representative sample, the average difference between the chains must be the same as the average difference within the chains. The plot in panel (b) presents the median and the $97.5 \%$ percentile indicating the confidence, being the approximate convergence diagnosed when the upper limit is close to 1 . If the value of the statistic is greater than 1.1, that is an indication of non-convergence (Gelman and Rubin, 1992; Kruschke, 2015).

From panel (c) of Fig. 4, the accuracy of the chain can be analyzed. An accurate chain generates a sample of sufficient size to obtain stable and accurate numerical estimates of the posterior distribution. Each step in the chain must provide independent information on the distribution of the parameter. However, when a very grouped chain is generated, the successive steps taken within the chain may not bring independent information. For this reason, it is necessary to measure the clustering of the chain. The less grouped it is, the more accurate the generated information is.

As the last stage in a full Bayesian inference, it should be checked whether the predictive model appropriately describes the observed data. Although the chain convergence may suggest that the posterior distributions produce correct estimates and represent the uncertainty, it is not usually known to which extent the model has adhered to the observed data, or even has an unknown systematic bias. To bridge this gap, it is necessary to conduct a PPC.

Comparisons between the simulations carried out via the PPC and the observed data are shown in Fig. 5, in which the temperatures around the transition region are selected to be shown. However, the check was performed for all temperatures to verify the goodness of the fitted model.

The PPC confirms that the model fits well the experimental (observed) data. It can be stated that the model successfully considers the uncertainties of the investigated VEM and supplies reliable information for estimating the dynamic properties of concern. 
(a)

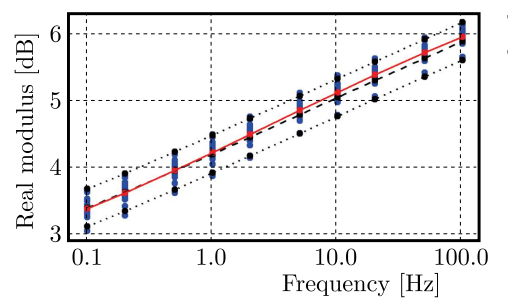

(b)
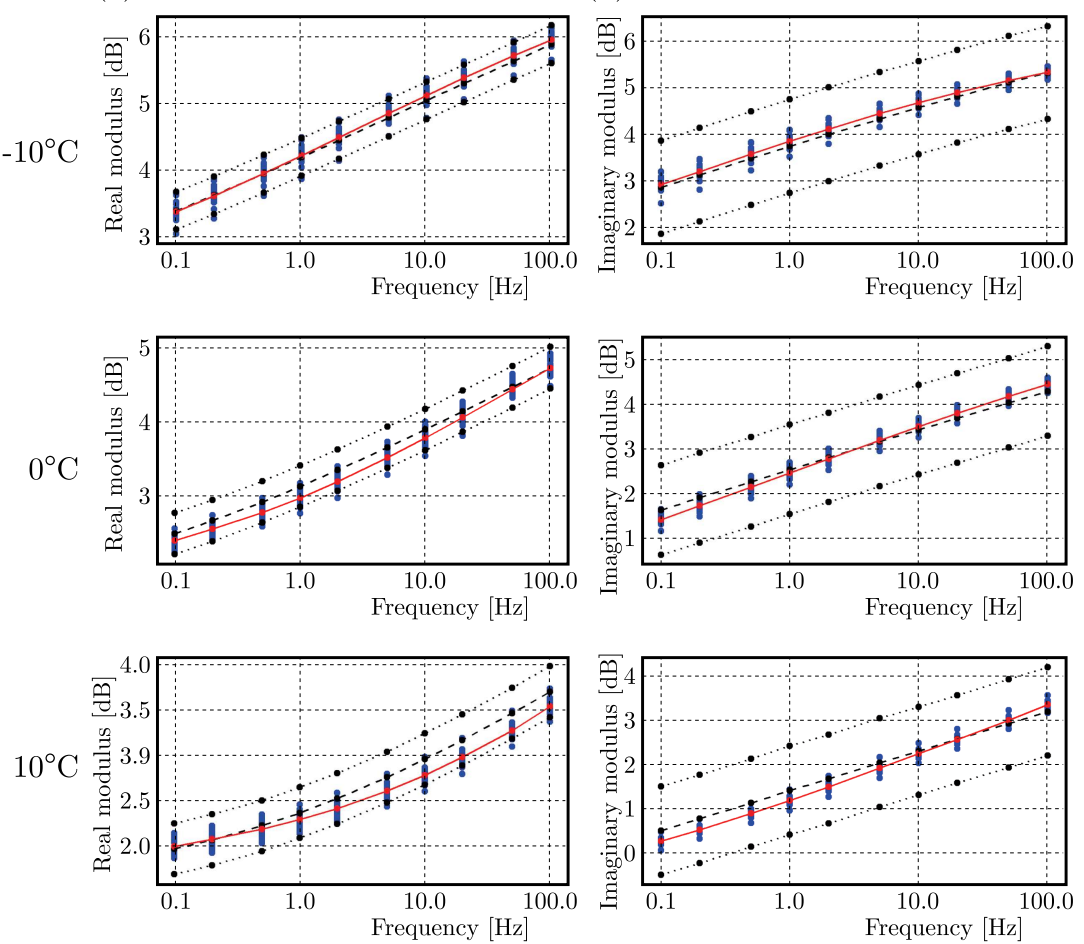

(c)
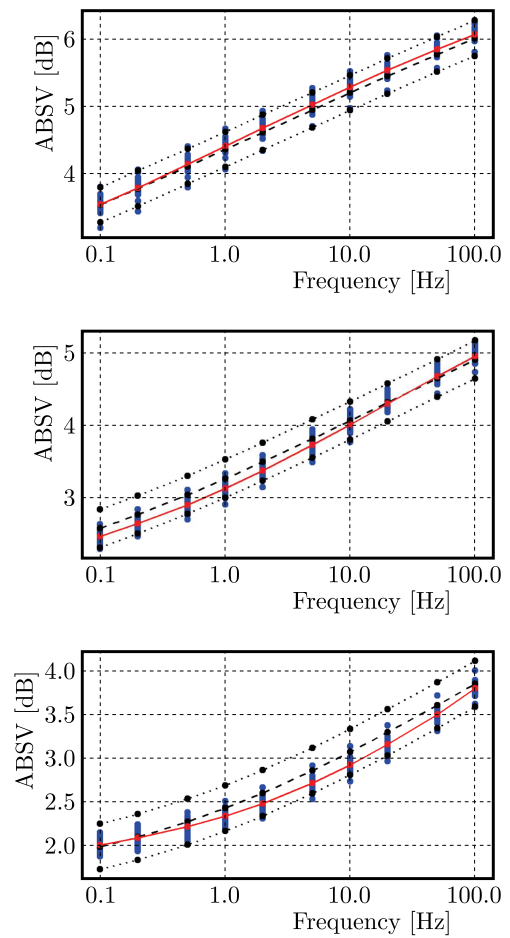

Fig. 5. Posterior predictive check - comparison between simulated and observed data for $-10^{\circ} \mathrm{C}, 0^{\circ} \mathrm{C}$ and $10^{\circ} \mathrm{C}$ : (a) real modulus, (b) imaginary modulus, (c) absolute value; $(-)$ simulated data mean, $(\cdots)$ percentile, $(-)$ observed data mean
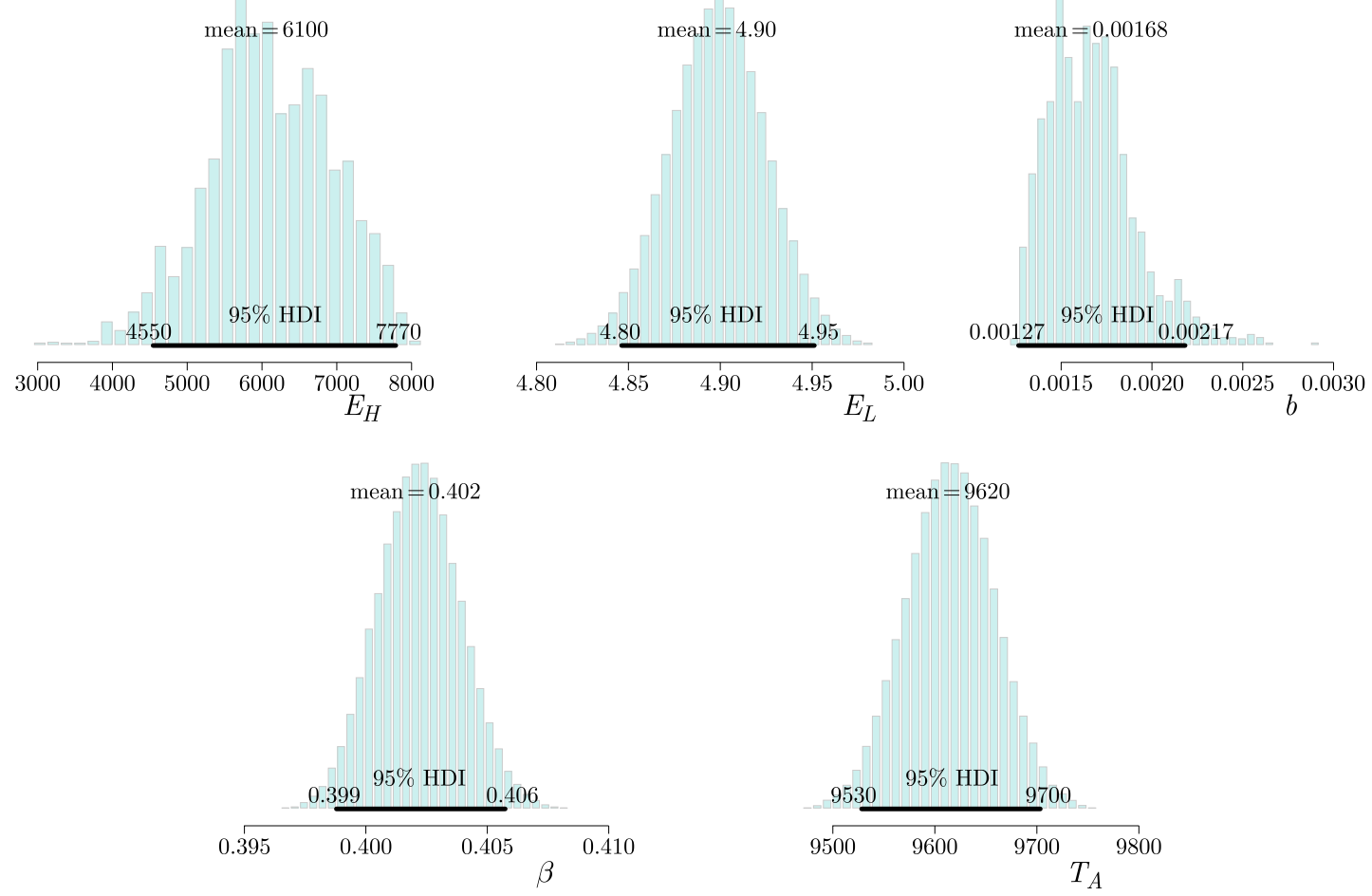

Fig. 6. Posterior probability density functions of the parameters excluding experimental data from $-40^{\circ} \mathrm{C}$ and $-20^{\circ} \mathrm{C}$ 
Aiming to estimate the posterior distribution for the same vector of material parameters using a narrower range of temperatures and examine if it is possible to achieve an accurate result, a new simulation was performed excluding the experimental data collected for lower temperatures, namely, $-40^{\circ} \mathrm{C}$ and $-20^{\circ} \mathrm{C}$. All the original rules were maintained, for instance, assigned prior distribution and likelihood function as well as the initial values. Figure 6 shows the posterior distribution density functions for each parameter, considering this new simulation.

The observed posterior distributions estimated from this set of data are different from the posterior distributions estimated for the set of data using the whole range of temperatures, especially with regard to parameters $E_{H}$ and $b$. However, to check the convergence of the simulation, it is important to perform the MCMC diagnostics, as mentioned above, so as to ensure that the chains have indeed converged to the posterior distributions. Figure 7 reproduces the same plots of Fig. 4, also for the parameter $E_{H}$, for sake of comparison.

(a)

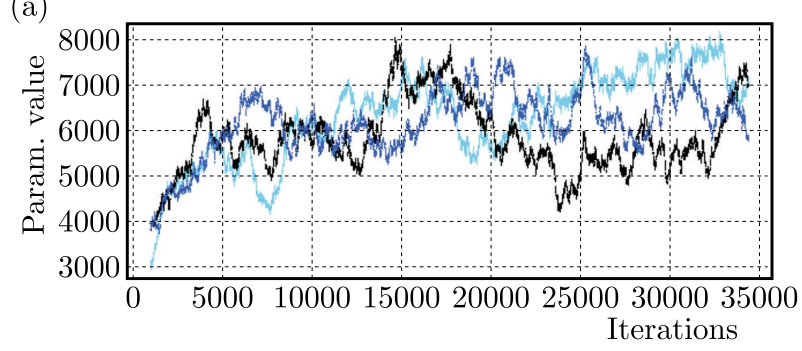

(c)

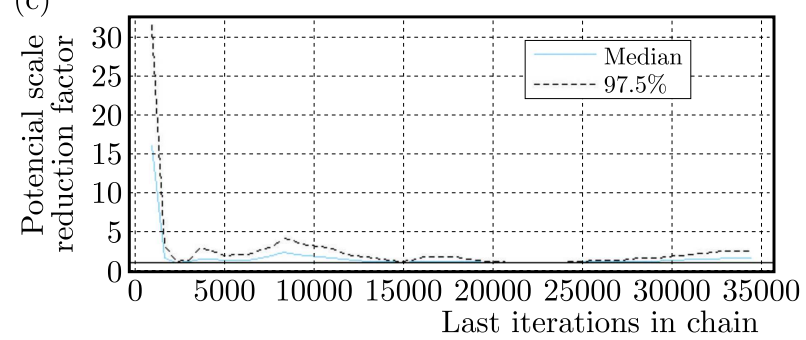

(b)

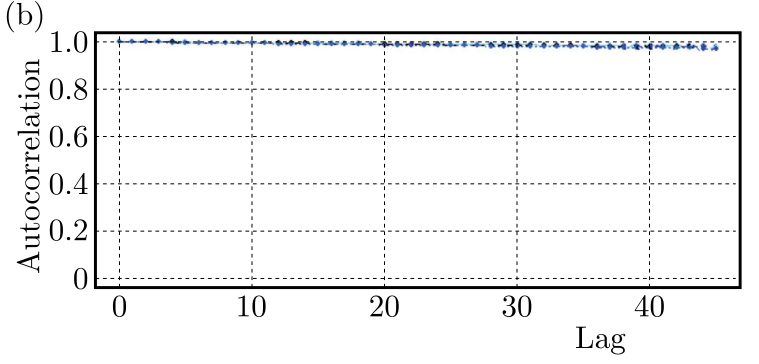

(d)

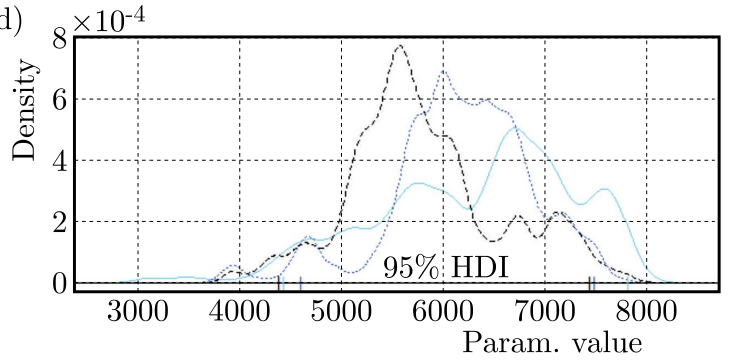

Fig. 7. Convergence diagnostics performed for the parameter $E_{H}$ excluding experimental data from $-40^{\circ} \mathrm{C}$ and $-20^{\circ} \mathrm{C}$ : (a) trace plot, (b) autocorrelation function, (c) Gelman-Rubin estimation,

(d) density plot

The trace plot in panel (a) shows a pattern of orphan chains, which is a clear indication of non-convergence. That is corroborated by the density plot, in panel (d), where the chains run in different regions of the parametric space indicating that the simulations do not produce representative values of the posterior distribution. The autocorrelation panel (b) also indicates that parameter values at successive chain steps do not provide independent information about the posterior distribution.

It can then be stated from the analysis of the MCMC convergence diagnostics that the corresponding probabilistic information is not reliable for use. Another simulation, additionally extracting the data related to temperature of $-10^{\circ} \mathrm{C}$, was also performed, leading to unreliable results as well. Those efforts point out to the understanding that the complete set of experimental data, contemplating a wide range of temperatures, is necessary to achieve a good fit of the model and leads to consistent estimation of the dynamic properties of the viscoelastic material. This understanding is termed herein as wide estimation of dynamic properties.

As pointed out earlier, a well-known and widely accepted method for identifying dynamic parameters of viscoelastic materials is the least-squares method. This method minimizes the least-squares error between experimental data and model predictions. According to Gogu et al. (2010), this method leads to reasonably accurate results. Nevertheless, by itself, it does not provide means of incorporating uncertainty as it is carried out by statistical inference. And 
although there are instances in which uncertainty may not be of primary concern, as observed in de Sousa et al. (2017), there are other instances where consideration of uncertainty is paramount.

Balbino (2012), who treated the same experimental data set used in this work, compared the analysis for the least-squares method with the frequentist inference approach. Frequentist inference is able to determine a dynamic parameter with a range of variation around the mean. The results are an indication that these parameters have a significant variation that could affect possible designs of vibration control devices using the viscoelastic material under investigation. However, from that approach, it is neither possible to characterize a probability distribution for each parameter nor to supply the outcome information for the future work as valuable prior information, as it is made available herein by the Bayesian inference approach.

It is of concern to obtain probability distributions of model parameters in order to probabilistically evaluate them over their entire domains. Those results also allow one to replicate the observed (measured) experimental data and compare the replicated set to the observed one. That is, actually, a more complete and suitable way of assessing the adequacy of the corresponding model, as carried out in the Posterior Predictive Check (PPC) (see Section 5.4).

In a companion paper (Préve et al., 2021), it is shown how the estimation produced by the Bayesian inference can be consistently employed to design and assess the performance of vibration control devices (namely, isolators and neutralizers). It is worth stressing that the same procedure followed to perform the PPC, described above, is applied there to generate simulated data.

\section{Conclusion}

Bayesian inference has been successfully applied in an unprecedented way in dynamic characterization of a typical viscoelastic material described by a four-parameter fractional order derivative model conjugated with the one-parameter Arrhenius equation. For this purpose, experimental data was obtained over wide ranges of frequency and temperature.

The Bayesian approach estimated the parameters of the dynamic properties of the viscoelastic material and incorporated sources of uncertainty by characterizing their posterior probability distribution functions. The Markov Chain Monte Carlo sampler performed simulations required for identification of the material parameters. A posterior predictive check was performed to analyze the goodness-of-fit of the model and it was shown to fit well the experimental data.

The main advantage of the Bayesian approach in models of this nature - clearly nonlinear is the ability to evaluate more realistically the uncertainties associated with the estimates and curves so obtained. It approximates - via MCMC - the posterior distribution of each parameter of concern.

The present work paves the way for the Bayesian approach to be employed in probabilistic dynamic characterization of viscoelastic materials in a wide range of frequencies and temperatures. In particular, it provides valuable information in efforts of the design and performance assessment of viscoelastic vibration control devices.

Acknowledgements

The authors would like to acknowledge CAPES, CNPq and UFPR for the financial support, as well as LACTEC for the valuable aid in this research work.

\section{References}

1. Bagley R.L., Torvik P.J., 1986, On the fractional calculus model of viscoelastic behavior, Journal of Rheology, 30, 133-156 
2. Balbino F.O., 2012, Statistical Analysis of Experimental Data on Dynamic Characterization of Viscoelastic Materials (in Portuguese), Master's Dissertation, Federal University of Paraná, Paraná, Brazil (available at http://www.prppg.ufpr.br/pgmec/wp-content/uploads/2016/05/ Tese_047_Fernanda-Oliveira-Balbino.pdf)

3. Gamerman D., Lopes H.F., 2006, Markov Chain Monte Carlo, 2nd ed., Chapman \& Hall

4. Gelman A., 2003, Bayesian formulation of exploratory data analysis and goodness-of-fit testing, International Statistical Review, 71, 2, 369-382

5. Gelman A., Rubin D.B., 1992, Inference from iterative simulation using multiple sequences, Statistical Science, 7, 4, 457-511

6. Gogu C., Haftka R., Le Riche R., Molimard L., Vautrin A., 2010, Introduction to the Bayesian approach applied to elastic constant identification, AIAA Journal, 48, 5, 893-903

7. Hernández W.P., Castello D.A., Roitman N., Magluta C., 2017, Thermorheologically simple materials: a Bayesian framework for model calibration and validation, Journal of Sound and Vibration, 402, 14-30

8. Hilton H.H., 2003, Optimum viscoelastic designer materials for minimizing failure probabilities during composite curing, Journal of Thermal Stresses, 26, 547-557

9. Jones D.I.G., 1992, Results of a round Robin test program: complex modulus properties of a polymeric damping material, Final Report for Period Oct 1986-May 1992, WL-TR-92-3104, Wright Laboratory, Flight Dynamics Directorate, Structural Dynamics Branch, Wright-Patterson AFB, Ohio, USA

10. Jones D.I.G., 2001, Handbook of Viscoelastic Vibration Damping, John Wiley \& Sons

11. Kruschke J.K., 2015, Doing Bayesian Data Analysis: A Tutorial with R, JAGS, and Stan, 2nd ed., Elsevier

12. Lopes E.M.O., 1998, On the Experimental Response Reanalysis of Structures with Elastomeric Materials, PhD Thesis, University of Wales, Cardiff, United Kingdom (available on request from either Arts and Social Studies Library, Cardiff University, or the last author)

13. Mead D.J., 1999, Passive Vibration Control, John Wiley \& Sons

14. Medeiros W.B.J., Préve C.T., Balbino F.O., da Silva T.A., Lopes E.M.O., 2019, On an integrated dynamic characterization of viscoelastic materials by fractional derivative and GHM models, Latin American Journal of Solids and Structures, 16, 1-19

15. Migon H.S., Gamerman D., Louzada F., 2015, Statistical Inference: An Integrated Approach, 2nd ed., CRC Press

16. Nashif A.D., Jones D.I.G., Henderson J.P., 1985, Vibration Damping, John Wiley \& Sons

17. NetzSCH, 2015, Dynamic-Mechanical Analysis DMA 242 C (available at http://photos.labwrench.com/equipmentManuals/7511-2751.pdf, accessed on 20 Feb 2021)

18. Plummer M., 2003, JAGS: A program for analysis of Bayesian graphical models using Gibbs sampling, Proceedings of the 3rd International Workshop on Distributed Statistical Computing, Vienna, Austria, 124, 125.10, 1-10

19. Plummer M., 2014, RJAGS: Bayesian Graphical Models Using MCMC, R package version 3-14, https://cran.r-project.org/web/packages/rjags/rjags.pdf

20. Préve C.T., Balbino F.O., Ribeiro Jr. P.J., Lopes E.M.O., 2021, On the use of viscoelastic materials characterized by Bayesian inference in vibration control, Journal of Theoretical and Applied Mechanics, 59, 3, 385-399

21. Pritz T., 1996, Analysis of four-parameter fractional derivative model of real solid materials, Journal of Sound and Vibration, 195, 103-115

22. R Core Team, 2015, R: A language and environment for statistical computing, R Foundation for Statistical Computing, Vienna, Austria. http://www.R-project.org/ 
23. Scherbakov M., Gurvich M.R., 2005, Probabilistic modeling of hysteretic behavior of elastomers under 3-D cyclic loading, Journal of Elastomers and Plastics, 37, 2, 123-147

24. Sousa T.L. De, Kanke F., Pereira J.T., Bavastri C.A., 2017, Property identification of viscoelastic solid materials in nomograms using optimization techniques, Journal of Theoretical and Applied Mechanics, 55, 1285-1297

25. Zhang E., Chazot J.D., Antoni J., Hamdi M., 2013, Bayesian characterization of Young's modulus of viscoelastic materials in laminated structures, Journal of Sound and Vibration, 332, 3654-3666

Manuscript received November 30, 2020; accepted for print March 29, 2021 